\title{
A Result Analysis of Translation Techniques of English to Hindi Online Translation Systems
}

\author{
Ekta Gupta \\ Student \\ Samrat Ashok Technology Institute \\ Vidisha (M.P.) India
}

\author{
Shailendra Kumar Shrivastava, PhD \\ Associate Prof. \\ Samrat Ashok Technology Institute \\ Vidisha (M.P.) India
}

\begin{abstract}
If the term "translation process" makes we consider of dictionaries, grammar rules, and debates about linguistic details, we're absolutely not alone. However, the translation process does not begin or end with transferring information from one language into another. In developing countries like Asian country and India where English is mainly half-hour recognize there automatic computational linguistics systems in education, analysis and industrial activities of very necessary role. Asian country has state a large assembly in Hindi is that the language you speak and in a very range of areas it works in all types of study and official. These days many on-line translator technologies use fully different computational linguistics approach. Like every translation approaches fully different characteristics, the result of the explanation would take issue. The purpose of this study is to create understanding about the different performance of the two online translation services due to the same actions they have. The experiment designed is meant show how the two online translation services have its have advantages and drawbacks which can affect their performance.
\end{abstract}

\section{Keywords}

Translation Quality Analysis, Translation Quality of Online Translation System for English to Hindi Translation.

\section{INTRODUCTION}

Machine Translation mainly deals with transformation of one language to another. Coming to the MT scenarios in India, it has huge scope due to many local languages of India. It is pertinent hat majority of the population in India are flowing in local languages such as Hindi, Punjabi etc. Given such a scenario, MT can be used to provide an interface of regional language. Machine translation is that the name for processed methods that automatize all or a section of the strategy of translating from one language to a unique. Throughout a huge multilingual society like India, there is great require for translation of documents from single language to a different language. As most of the higher study material, research journals and different commonplace communication tools are in English; these materials are to be translated into Hindi or particular local languages to possess an applicable higher study and communication with the individuals.

Machine Translation is a sub-field of computational linguistic that investigates the use of computer software to translate copy or speech from single natural language to another. At its basic level, MT performs simple exchange of words in single natural language for words in another. Current machine translation software often allows for customization by field or profession, improving output by limiting the scope of allowable substitutions. This technique is mainly effective in domain where formal or standard language is used it follows that machine translation of government and authorized documents more willingly produces usable output than conversation or less standardized text. Advanced study within the field of artificial intelligence and Computational Linguistics created a promising development of translation technology. This helped within the development of usable computational linguistics Systems in certain well-defined domains. Totally prime quality automatic computational linguistics system to induce could be a difficult task several organizations like Google, Microsoft, IBM, and lots of different etc. are engaged in development of MT systems. The technology is success new heights, right from idea of ideas up to the sensible implementation. It's necessary, that equal emphasis is place to remove the language divide that causes communication gap among totally different sections of societies. Natural language process (NLP) is that the field that strives to fill this gap. Machine translation (MT) principally deals with transformation of 1 language to a different. Coming back to the MT scenarios in Republic of India, it's huge scope because of several regional languages like Hindi, Punjabi etc. MT will be used to offer an interface of regional language.

\section{THEORY}

Machine Translation system are required to translate literary works that from any language into native languages. The literary work is fed to the MT system and translation is completed. Such MT system will break the language barriers by creating offered work rich sources of literature available to people across the globe.

MT additionally overcomes the technological barrier. Most of the data offered is in English that is known by only 3rd of the population. This has led to digital divide within which only small section of society will perceive the content presented in digital format. MT will facilitate during this regard to overcome the digital divide.

The following are four kinds of machine translation system:

\section{MT for Watcher (MT-W)}

MT for watcher is meant for readers WHO needed to achieve access to some data written in foreign language WHO also are ready to accept potential bad 'rough' translation instead of nothing. This was the kind of MT envisaged by the pioneers. This came in with the requirement to translate military technological documents.

\section{MT for revisers (MT-R)}

MT for revisers aims at producing raw translation mechanically with a high quality with a high quality similar to that of the primary drafts made by human. the translation output may be thought of only as brush-up so the professional translator may be free of that boring and time consuming task. 


\section{MT for translators (MT-T)}

MT for translator's aims at serving to human translators do their job by providing on-line dictionaries, synonym finder and translation memory. this kind of machine translation system is sometimes incorporated into the translation work stations and also the computer based translation tools.

\section{MT for Authors (MT-A)}

MT for authors aims at authors desperate to have their texts translated into one or many languages and acceptive to write under control of the system or to help the system clear up the vocalization so satisfactory may be obtained without any revision.

\section{METHOD}

\subsection{Statistical Machine Translation}

In this the input is considered as a distorted version of the target language sentence and the task is to find the most likely source language sentence giving the translation.

The task involves three steps:

1. Estimating the language probability $\mathrm{P}(\mathrm{t})$

2. Estimating the translational model probability $\mathrm{p}(\mathrm{s} / \mathrm{t})$

3. Devising an efficient search for the target text that maximizes their product.

We have to find the sentence $\mathrm{T}$ for which $\mathrm{p}(\mathrm{s} t)$ is maximum.

$\mathrm{P}(\mathrm{s}, \mathrm{t})=\arg \max \mathrm{p}(\mathrm{s}, \mathrm{t})=\arg \max \mathrm{p}(\mathrm{t}) \mathrm{p}(\mathrm{s} / \mathrm{t})$

In the above model , $\mathrm{s}^{\text {ec }}$ is the source language sentence and „te is the target language sentence. The probabilities are to be calculated from the parallel corpus. Smoothing techniques are required for handling data sparsity problem that occurs in any noisy channel model.

\subsection{Example based Machine translation system}

An Example based Machine translation system (EBMT) system maintains a corpus consisting of translation examples between source and target languages. An EBMT system has two modules: Retrieval module and an adaptation module. The retrieval module retrieves a similar sentence and its translation from the corpus for the given source sentence. The adaptation module then adapts the retrieved translation to get the final corrected translation.

Consider the English to Hindi translation for the following sentence.

"Rama sings a song"

The retrieval module retrieves the following sentence and its translation from the corpus from a list of approximately matching sentences. It uses some similarity measures based on word similarity or syntactic and semantic similarity to identify this set of approximately matching sentences. From these the system selects the sentence with closest match with the input sentence.

If the system selects "Rohit sings a song" and its translation "Rohit geet gaata hai" as the closest one, it replaces Rohit with Rama and gaata with gaathi and finally forms the translation.

"Rama geet gaati hai".

Here the adaptation is required to replace the word and suffix replacements. This method may not work in case of translation divergence where structurally similar sentences of the source language get translated into a different structure.

\subsection{Language model}

A language model gives the probability of a sentence. The probability is computed using n-gram model. Language model can be considered as computation of the probability of single word given all of the words that precede it in a sentence. The goal of statistical machine translation is to estimate the probability of a sentence. A sentence is decomposed in to the product of conditional probability.

By using chain rule, this is made possible as shown in equation 2 . The probability of sentence $\mathrm{P}(\mathrm{S})$, is broken down as the probability of individual words $\mathrm{P}(\mathrm{W})$.

$$
\begin{aligned}
P(S) & =P\left(W_{1}, W_{2}, W_{3}, \ldots \ldots \ldots \ldots . ., W_{n}\right) \\
& \left.=P\left(W_{1}\right) P\left(W_{2} \mid W_{1}\right) P\left(W_{3} \mid W_{1} W_{2}\right) P\left(W_{4} \mid W_{1} W_{2} W_{3}\right) \ldots . P\left(W_{n} \mid W_{1} W_{2} \ldots W_{n-1}\right)\right)
\end{aligned}
$$

In order to calculate sentence probability, it is required to calculate the probability of a word, given the sequence of word preceding it. An n-gram model simplifies the task by approximating the probability of a word given all the previous words.

An $n$-gram of size 1 is referred to as a unigram; size 2 is a bigram (or, less commonly, a digram); size 3 is a trigram; size 4 is four-gram and size 5 or more is simply called a n-gram.

\section{RESULT}

This is algorithm which convert file to text of data

//create file inputstream object to read data from file

FileInputStream fs=new FileInputStream(src);

//create document object to wrap the file inputstream object

XWPFDocument docx=new XWPFDocument(fs);

//create text extractor object to extract text from the document

XWPFWordExtractor extractor $=$ new

XWPFWordExtractor(docx);

//create file writer object to write text to the output file

FileWriter fw=new FileWriter(desc);

//write text to the output file

fw.write(extractor.getText());

//clear data from memory

fw.flush();

//close inputstream and file writer

fs.close();

fw.close();

\}catch(IOException e) \{e.printStackTrace();\}\} 


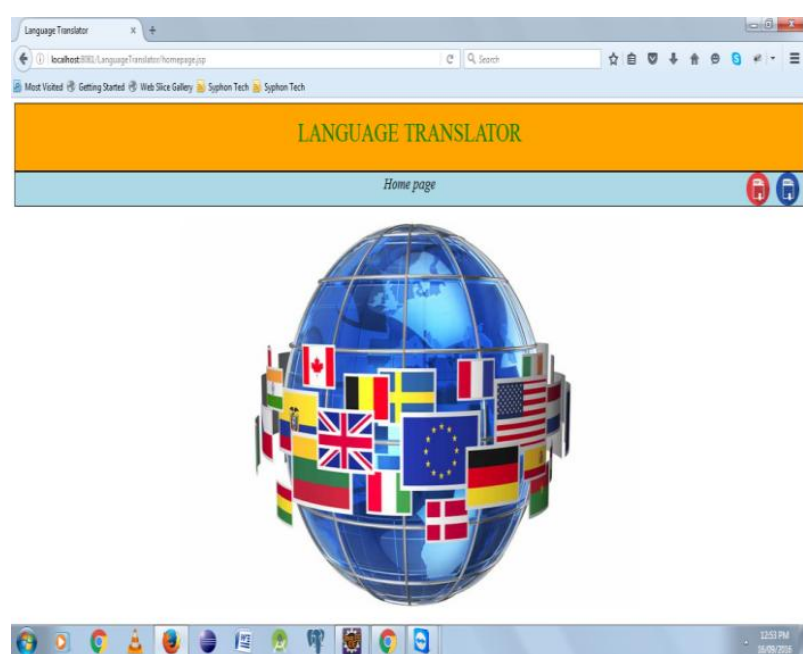

Fig:1 Translator Home
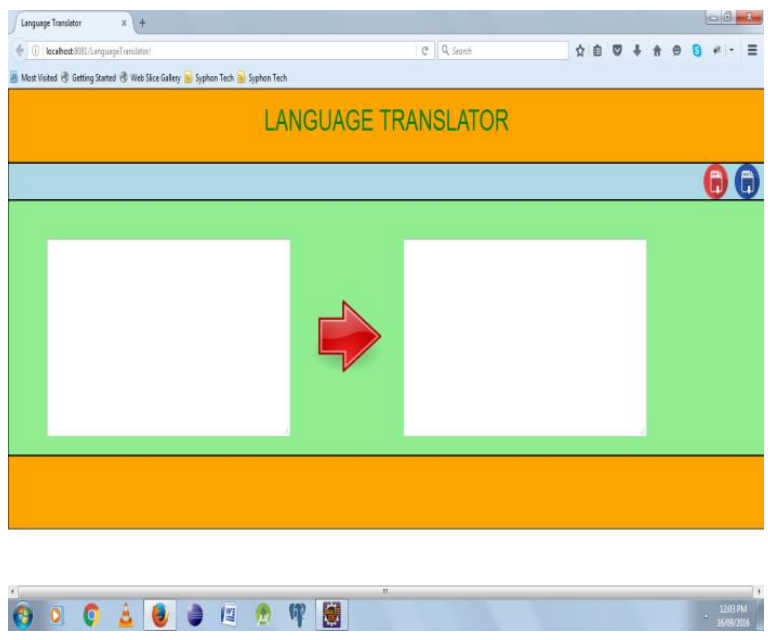

Fig: 2Translator layout section

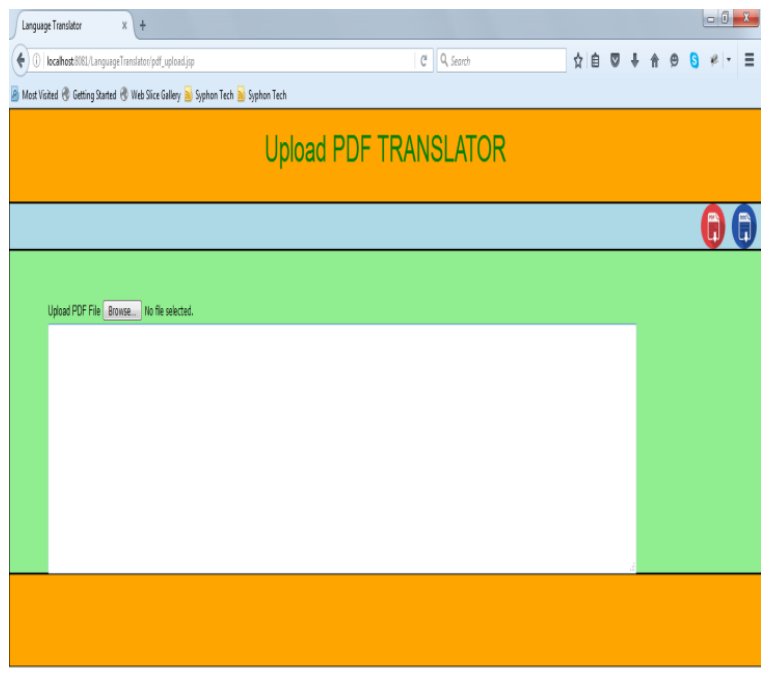

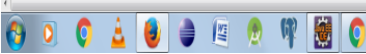

Fig:3 Translator File selection

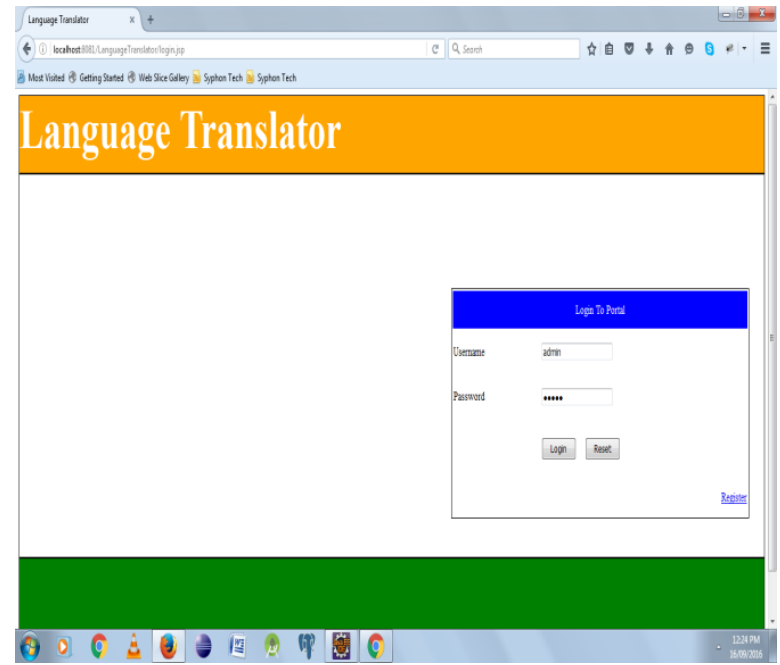

Fig:4 Translator Home

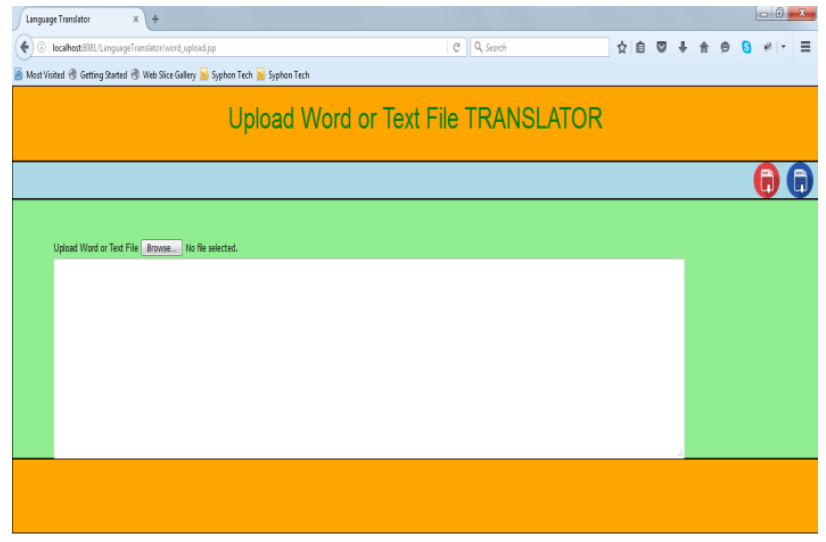

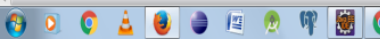

Fig: 5 Translator layout section

\section{CONCLUSION}

In this paper result analysis of Translation Techniques of English to Hindi Online Translation Systems which provides above results, that shows the complication of the previous ways ought to be increased. Our results show that the planned techniques considerably improve the translation quality. The performance of the two is also different and this has been proven in this study. I chose Google Translate and Bing Translator to represent English to Hindi translation. In this studies it is found that Bing Translator did a better job when translating English to Hindi and vice versa.

\section{REFERENCES}

[1] Bhojraj singh dhakar, sitesh kumar sinha, krishna kumar pandey "A Survey of Translation Quality of English to Hindi Online Translation Systems (Google and Bing)", International Journal of Scientific and Research Publications, Volume 3, Issue 1, January 2013

[2] Kanika Gupta, Monojit Choudhury, Kalika Bali "Mining Hindi-English Transliteration pair from OnlineHindiLyrics"http://www.google.com/intl/en/press/ zeitgeist2010/regions/ in.html

[3] Sanjay Dwivedi and Pramod Sukhadeve "Translation Rules for English to Hindi Machine Translation System: 
Homoeopathy Domain", The International Arab Journal of Information Technology, Vol. 12, No. 6A, 2015

[4] Atul Kr. Johan, Akanksha Bansal, Sumedh Hadke and Girish Nath Jha "Evaluation of Hindi-English MT Systems", http://translate.google.co.in/

[5] Prashant Mathur "Automatic Translation of Noun Compounds from English to Hindi", Language Technology Research Center International Institute of Information Technology Hyderabad - 500032, INDIA October, 2011

[6] Akshar Bharati, Amba Kulkarni" English from Hindi viewpoint: A Paaninian Perspective" Satyam Computer Services Limited, [Presented at Platinum Jubilee conference of Linguistic Society of India, held at CALTS, University of Hyderabad, Hyderabad, during 6th8th [Dec 2005]

[7] R.M.K. Sinha , A. Jain "An English to Hindi MachineAided Translation System" Indian Institute of Technology, Kanpur, India.

[8] Kunal Sachdeva, Rishabh Srivastava, Sambhav Jain, Dipti Misra Sharma "Hindi to English Machine Translation:Using Effective Selection in Multi-Model SMT'Language Technologies Research Center, International Institute of Information Technology, Hyderabad.

[9] B. Hettige, A. S. Karunananda "Developing Lexicon Databases for English to Sinhala Machine Translation", University of Sri Jayewardenepura, Sri Lanka

[10] Latha R. Nair David Peter S, "Machine translation systems for Indian language", IJCA Journal 2012, Volume 39 - Number 1.

[11] Nisheeth Joshi, Iti Mathur "Design of English-Hindi Translation Memory for Efficient Translation", National Conference on Recent Advances of Computer Engineering, 2011, Jaipur, India.

[12] Aditi Kalyani, Hemant Kumud, Shashi Pal Singh "Assessing the Quality of MT Systems for Hindi to English Translation", International Journal of Computer Applications (0975 - 8887)Volume 89 - No 15, March 2014.

[13] Charles Schafer, David Smith "An Overview of Statistical Machine Translation" [Johns Hopkins University] http://www.nist.gov/speech/tests/mt" Automatic Evaluation of Machine Translation Quality Using N-gram Co-Occurrence Statistics"

[14] Jonathan H. Clark Chris Dyer Alon Lavie Noah A. Smith "Better Hypothesis Testing for Statistical Machine Translation: Controlling for Optimizer Instability "[Language Technologies Institute Carnegie Mellon University Pittsburgh, PA 15213, USA]

[15] Kishore Papineni, Salim Roukos, ToddWard, andWeiJing Zhu. 2002. Bleu: a method for automatic evaluation of machine translation. In Proc. of the 40th Annual Meeting of the Association for Computational Linguistics (ACL), pages 311-318, Philadelphia, PA, July.

[16] LDC. 2005. Linguistic data consortium Chinese training data resources.
[17] http://www.ldc.upenn.edu/Projects/TIDES/mt2005cn.ht $\mathrm{m}$

[18] Malcolm Williams "The Application of Argumentation Theory to Translation Quality Assessment"

[19] Mary Hearne, Andy Way" Statistical Machine Translation: A Guide for Linguists and translator "[School of Computing, Dublin City University].

[20] Rafal S Uzar "A corpus methodology for analyzing translation "[University of Lodz]

[21] Riccardo Schiaffino, Franco Zearo "Translation Quality Measurement in Practice", 46th ATA Conference, Seattle. Aliquantum ..., 2005

[22] G V Garje and G K Kharate "survey of machine translation systems India", International Journal on Natural Language Computing (IJNLC) Vol. 2, No.4, October2013.

[23] Yongwei Yang, James Harter, Eldin J. Ehrlich "A Methodological Analysis of Translation Quality", Journal of Cross-Cultural Psychology, Vol. 37 No. 5, September 2006

[24] B. Hettige, A. S. Karunananda "Developing Lexicon Databases for English to Sinhala Machine Translation", University of Sri Jayewardenepura, Sri Lanka.

[25] Niladri Chatterjeea, Anish Johnsonb, Madhav Krishnab "Some Improvements over the BLEU Metric for Measuring Translation Quality for Hindi”, Indian Institute of Technology, Hauz Khas, New Delhi.

[26] Neeraj Tomer, Deepa Sinha "Evaluating Machine Translation Evaluation's BLEU Metric for English to Hindi Language Machine Translation", Volume 1, No. 6, August 2012.

[27] Nakul harma, Prateek Bhatia "English to Hindi Statistical Manchine Translation System”Thapar University Patiala june 2011.

[28] Sukhadeve P. and Dwivedi S., "Developing Hindi POS Tagger for homoeopathy Clinical language," in Proceedings of the 2nd International Conference Advances in Computer Science and Information Technology, Bangalore, India, pp. 310-316, 2012.

[29] Sukhadeve P. and Dwivedi S., "Enlargement of Clinical Stemmer in Hindi Language of Homoeopathy Province," in Proceedings of the 2nd International Conference Advances in Computer Science and Information Technology, Bangalore, India, pp. 239-248, 2012.

[30] The Stanford Natural Language Processing Group., available at: http://nlp.stanford.edu/software/tagger.html, last visited 2013.

[31] Unnikrishnan P., Antony P., and Soman K., "A Novel Approach for English to South Dravidian Language Statistical Machine Translation System," the International Journal on Computer Science and Engineering, vol. 2, no. 8, pp. 2749- 2759, 2010. 